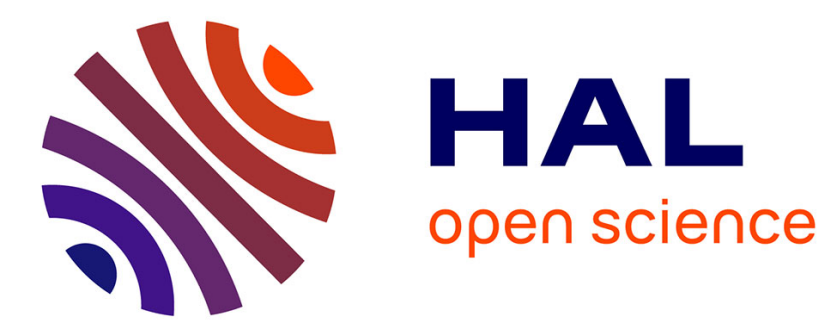

\title{
Réseaux 2d aléatoires à nanotubes de carbone Jean Christophe Gabriel
}

\section{To cite this version:}

Jean Christophe Gabriel. Réseaux 2d aléatoires à nanotubes de carbone. Comptes Rendus. Physique, 2010, 11, pp.362 - 374. 10.1016/j.crhy.2010.07.016 . cea-01549116

\section{HAL Id: cea-01549116 https://hal-cea.archives-ouvertes.fr/cea-01549116}

Submitted on 28 Jun 2017

HAL is a multi-disciplinary open access archive for the deposit and dissemination of scientific research documents, whether they are published or not. The documents may come from teaching and research institutions in France or abroad, or from public or private research centers.
L'archive ouverte pluridisciplinaire HAL, est destinée au dépôt et à la diffusion de documents scientifiques de niveau recherche, publiés ou non, émanant des établissements d'enseignement et de recherche français ou étrangers, des laboratoires publics ou privés. 
Propriétés optiques des nanotubes / Optical properties of nanotubes

\title{
Réseaux 2d aléatoires à nanotubes de carbone
}

\section{$2 d$ Random networks of carbon nanotubes}

\author{
Jean-Christophe P. Gabriel \\ DSM/DPNS, CEA, 17, rue des Martyrs, 38054 Grenoble cedex, France
}

\section{N F O O A R T I C}

\section{Mots-clés :}

Nanotube

Carbone

Réseau

Synthèse

Propriétés

Simulation

Intégration

Application

Industriels

Brevets

\section{Keywords:}

Nanotube

Carbon

Network

Synthesis

Property

Simulation

Integration

Application

Industries

Patents

\begin{abstract}
R É S U M É
L'utilisation de nanotubes de carbone dans des applications électroniques se heurte au problème du contrôle de leur chiralité. Très tôt les chercheurs et industriels ont contournés cet écueil avec une approche statistique et l'utilisation de réseaux aléatoires de nanotubes qui permettent de moyenner leurs différentes propriétés. Après un rappel de l'historique du sujet, cet article se concentre sur les développements très rapides et récents du domaine.
\end{abstract}

(C) 2010 Académie des sciences. Publié par Elsevier Masson SAS. Tous droits réservés.

\section{A B S T R A C T}

A major challenge in the use of carbon nanotubes in electronic devices can be found in the problem of controlling their chirality. Very early, researchers, both from academia and industry, have circumvented the problem using a statistical averaging of the nanotube properties and the use of a random network. After a rapid review of the historical background of this subject, the article will focus on recent (up to 2008) and rapidly progressing results.

(C) 2010 Académie des sciences. Publié par Elsevier Masson SAS. Tous droits réservés.

\section{Mise en oeuvre}

\subsection{Travaux préliminaires}

Suite à la re-découverte [1] des nanotubes de carbone multi-parois (MWNT) par lijima [2], mono-paroi par Bethune [3] et à la publication du premier transistor à effet de champ à base de nanotube de carbone [4], de nombreux travaux ont été effectués sur des composants à base d'un ou quelques nanotubes de carbone. Les premiers réseaux $2 \mathrm{~d}$ aléatoires, hors buckypaper, ont ensuite été publiés par le groupe de Hongjie Dai à l'Université de Stanford mais ces derniers étaient obtenus par croissance CVD directement sur une couche épaisse de catalyseur fait d'aluminosilicate poreux déposé sur un

Adresse e-mail:Jean-Christophe.Gabriel@cea.fr. 
a)

1)

2)

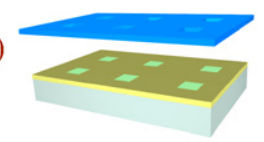

3)

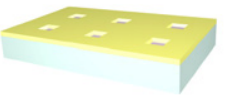

c)

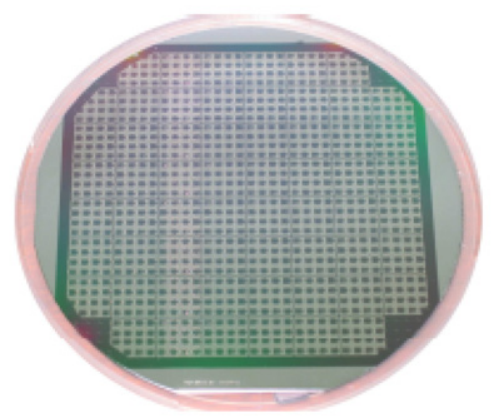

4)

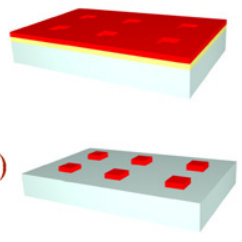

6)

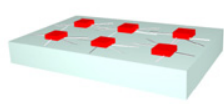

b)

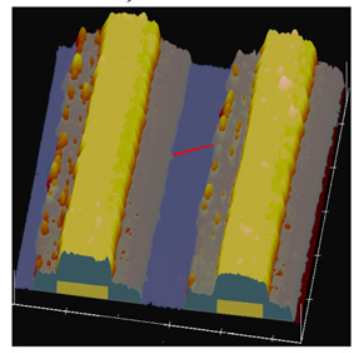

d)

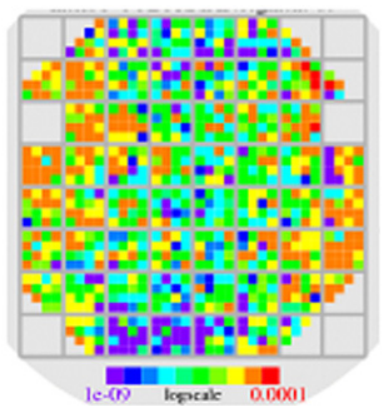

Fig. 1. a) Procédé de fabrication de transistors à nanotube unique utilisant la réalisation d'îlots de catalyseurs par lithographie suivie d'une croissance par CVD; b) Image AFM d'un nanocompsant à SWNT avec passivation des contacts; c) Wafer $100 \mathrm{~mm}$ obtenu à Nanomix selon ce procédé en septembre 2002 et; d) Wafer map en conductance montrant une conductance variant sur plusieurs ordres de grandeurs.

Fig. 1. a) Fabrication process of single nanotube transistors using the deposition of catalyser islands by lithography followed by CVD; b) AFM image of a SWNT nanocomponent with contact passivation; c) A $100 \mathrm{~mm}$ wafer obtained at Nanomix using this procedure in September 2002; and d) A map of the wafer conductance showing the conductance varying over several orders of magnitude.

substrat à l'aide d'une tournette [5]. De premières caractérisations électriques furent néanmoins publiées grâce au dépôt de contacts d'or directement sur le catalyseur. Ce procédé n'était cependant pas compatible avec la production à grande échelle de composant électronique [6].

La croissance des nanotubes par procédé CVD n'est et n'ai toujours pas adapté pour la production industrielle de nanocomposants à SWNT unique du fait du manque de contrôle de cette méthode. Notamment cette méthode ne permet pas la sélection de la chiralité des nanotubes synthétisés et de ce fait une grande polydispersité des distributions en conductance, transconductance et modulation des transistors obtenus est observée (Fig. 1).

\subsection{Les premiers travaux}

Du fait de la nécessité de produire, à grande échelle et à de bons rendements, des senseurs électroniques à base de CNT, le besoin de moyenner les propriétés des SWNTs est rapidement apparu comme une bonne solution à l'équipe de recherche de la startup Nanomix que j'avais rejoint en 2001 quelques mois après son financement initial (septembre 2000). La solution d'utiliser de nombreux SWNTs contactant la source et le drain d'un transistor en configuration parallèle a tout d'abord été étudiée mais n'était pas entièrement satisfaisante car la modulation des transistors obtenus n'était pas satisfaisante du fait de la croissance indifférenciée de tubes semiconducteurs et métalliques. Cette modulation pouvait certes être améliorée en détruisant les nanotubes conducteurs, par la méthode publiée par le groupe d'Avouris à IBM [7], mais ce procédé est difficile à mettre en place dans une ligne de production à l'échelle du wafer sur des dizaines de milliers de transistors. Nous avons ainsi été amenés à étudier très tôt les réseaux $2 d$ aléatoires. La première méthode développée consistait tout simplement à soumettre à un plasma d'oxygène intense des îlots de catalyseurs. Sous l'effet du plasma, le catalyseur était projeté sous forme de nanoparticules sur l'ensemble du wafer. Par rapport à une calcination classique à haute température, ce procédé de calcination du catalyseur permettait d'éviter la diffusion des métaux catalytiques dans la couche de $\mathrm{SiO}_{2}$ située en dessous du catalyseur et servant de diélectrique au transistor à grille arrière. Cette diffusion est un problème car elle peut engendrer des courants de fuite importants. Les FETs obtenus à partir de ces réseaux aléatoires ont permis l'obtention d'une très bonne reproductibilité des transistors ainsi que de leur modulation $(50<$ Mod $<200)$ et ce à l'échelle des wafers $100 \mathrm{~mm}$ sur lesquels nous développions le procédé d'intégration. Ces travaux ont fait l'objet d'un dépôt de brevet en juin 2002 (Fig. 2) [8,9]. 


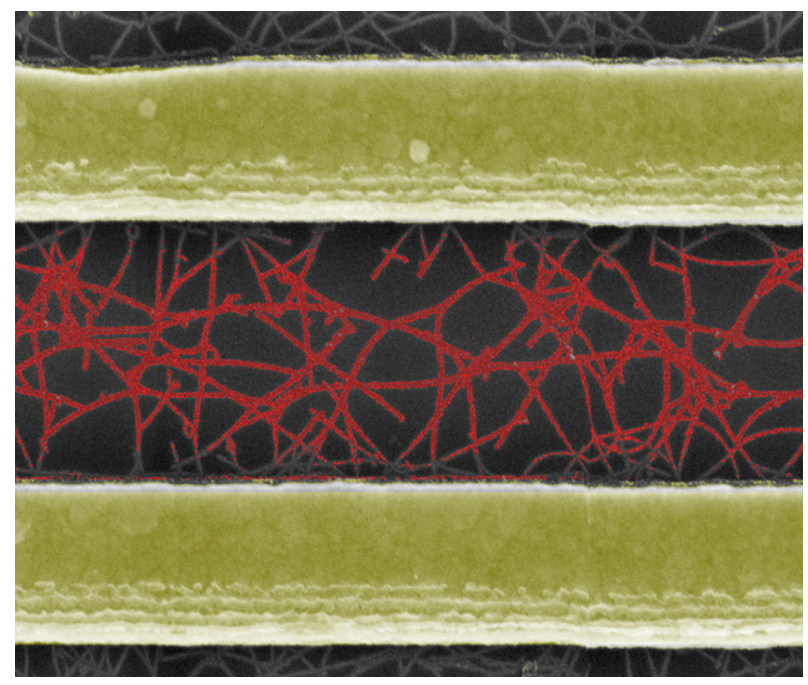

Fig. 2. Image de microscopie électronique à balayage (couleurs artificielles) d'un réseau aléatoire de nanotubes (rouge) entre deux contact métalliques (en jaune) obtenu à Nanomix en 2002. Extrait avec la permission de Nanomix.

Fig. 2. SEM image in false colours of random network of nanotubes (red) between metla contacts (yellow) made at Nanomix in 2002. Published with permission of Nanomix.

De façon indépendante à ces travaux, le groupe d'Eric S. Snow au Naval Research Laboratory a aussi travaillé sur de tels transistors, produits sur de petits fragments de wafers. La croissance se faisait à partir d'un catalyseur déposé par tournette directement sur le wafer. La demande de brevet de Nanomix sur le sujet n'étant pas encore publique, c'est le papier publié par ce groupe en mars 2003 qui a révélé à la communauté scientifique la faisabilité de FETs en utilisant ces réseaux 2d aléatoires [10] ainsi que leur intérêt comme senseur chimique [11].

La valeur ajoutée des réseaux aléatoires de nanotubes vis-à-vis des structures à nombreux nanotubes parallèles pour la réalisation de transistors provient de la meilleure reproductibilité et valeur en conductance et en modulation des composants obtenus. Notamment et de façon empirique il a immédiatement été observé que la modulation des transistors s'améliore, en moyenne, avec l'augmentation de la distance entre la source et le drain. Trois composantes participent à cette amélioration. La première est que 2/3 des structures possibles de nanotubes ont la structure de bande d'un semi-conducteur. Ainsi, plus la distance entre la source et le drain est élevée par rapport à la longueur moyenne des nanotubes, plus la probabilité qu'un chemin de conduction formé uniquement de nanotube métallique est faible $\left(P=(1 / 3)^{n}\right.$ où $n$ est le nombre de tubes formant la junction entre le source et le drain). Deuxièmement, la jonction nanotube-nanotube n'est pas métallique. Enfin, la présence de défaut sur un nanotube métallique induit généralement l'ouverture d'un gap dans le diagramme de bande et ainsi rend le tube semiconducteur [12]. Enfin, la présence d'un seul défaut sur un SWNT est suffisante pour induire une transition vers un état semiconducteur, comme cela a été très joliment montré expérimentalement par Collins et al. à l'Université d'Irvine [13].

Notons enfin que les propriétés électroniques des FETs à réseau aléatoire de nanotubes de carbone ne cessent de s'améliorer. On peut notamment citer des travaux japonais qui ont utilisés des matériaux à haute constante diélectrique $\left(\mathrm{Ba}_{0.4} \mathrm{Sr}_{0.6} \mathrm{Ti}_{0.96} \mathrm{O}_{3}, \varepsilon_{s}=100\right)$ comme isolant de grille ce qui leur a permis de réduire de $90 \%$ le voltage de switch, avec un on/off ratio de $10^{5}$ ainsi qu'une réduction très significative de l'hystérésis [14]. L'hystérésis est en effet une composante importante des transistors à nanotubes sur $\mathrm{SiO}_{2}$. Elle est, entre autre, reliée à la présence de charges mobiles sur la surface sur laquelle sont posés les nanotubes. Ainsi, toute stratégie tendant à réduire ces charges ou leur mobilité induit une réduction de cette hystérésis [15]. Enfin, un atout supplémentaire de ces réseaux est qu'ils apparaissent comme des structures pouvant résister à de fortes irradiations [16].

\subsection{Les réseaux $2 d$ aléatoire sur substrat flexible transparent}

Une fois démontrée la possibilité de créer un transistor à effet de champ et grille arrière et à base d'un réseau aléatoire de SWNT fût démontrée, l'étape suivante était d'utiliser de tels réseaux sur un support flexible. Le premier transistor sur substrat flexible fut réalisé, publié et breveté par Nanomix en 2003 (Fig. 3) [17]. On notera au passage la nature transparente de ce composant. Ces composants furent simplement réalisés par le transfert du système réseau de CNT + contacts métalliques : (i) par le dépôt d'un film de polyimide sur le dispositif développé précédemment sur wafer de silicium par photolithographie; (ii) suivi d'une digestion du diélectrique $\mathrm{SiO}_{2}$ sous jacent par du HF dilué, qui permet de libérer le réseau de nanotubes contactés et imbriqués dans le polymère. Même si ces premiers composants présentaient des caractéristiques électroniques de piètre qualité, l'intérêt pour l'électronique flexible que représente ces réseaux aléatoires de SWNT vis-à-vis des molécules organiques ou organométalliques utilisées jusqu'alors dans les transistors organiques est la très haute mobi- 


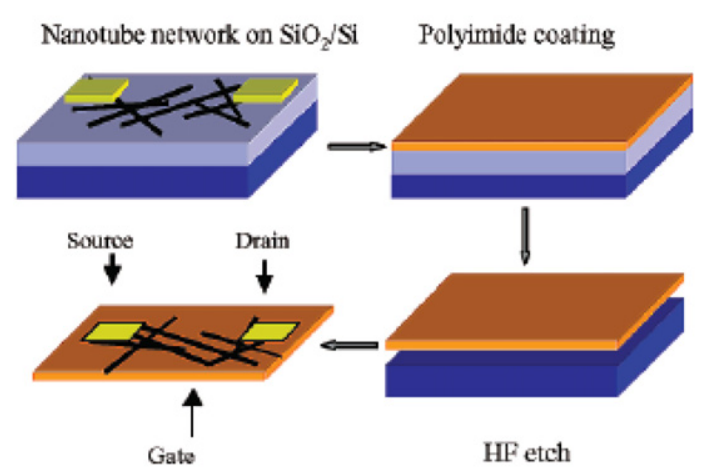

(a)

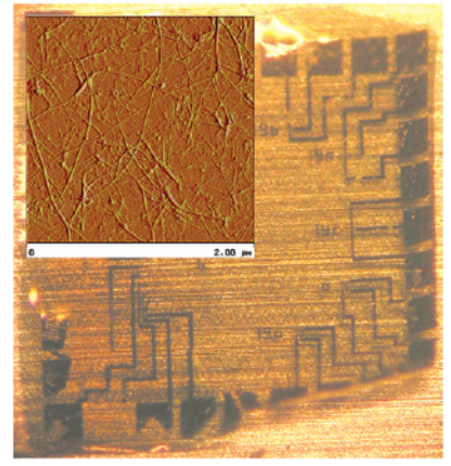

(b)

Fig. 3. a) Méthode de fabrication d'un transistor flexible; b) Photographie du dispositif réalisé avec en insertion image AFM du réseau aléatoire de SWNT avant transfert. Extrait avec la permission de Nanoletters 3 (10) (2003) 1353, Ref. [17a]. Copyright (2003) American Chemical Society.

Fig. 3. a) Fabrication method for a flexible transistor; b) Photograph of this with as insert an AFM image of the random network of SWNTs before transfer. Published with permission of Nanoletters 3 (10) (2003) 1353, Ref. [17a]. Copyright (2003) of the American Chemical Society.

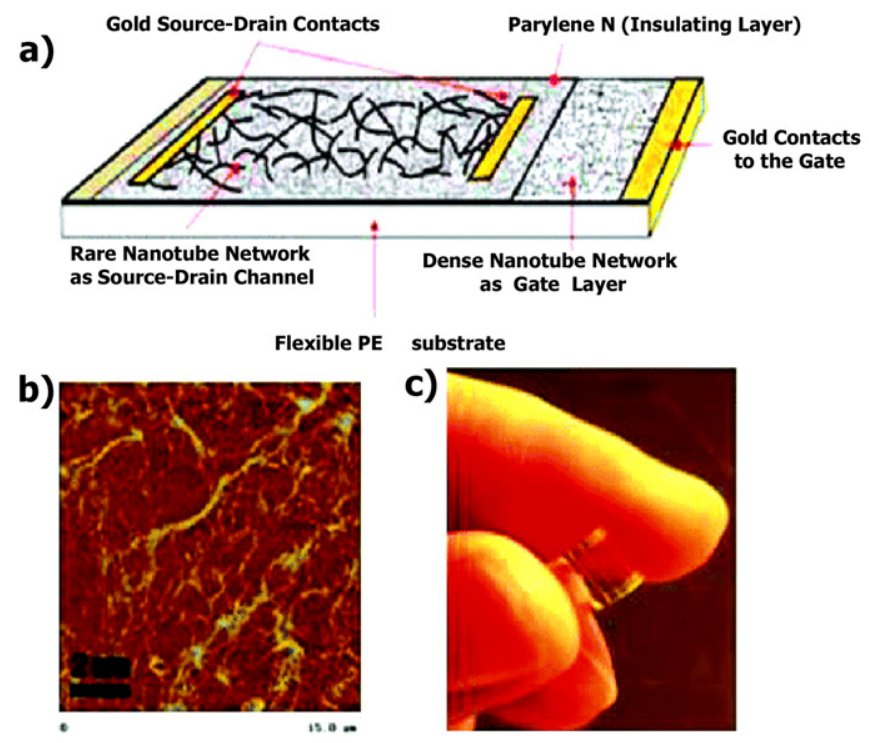

Fig. 4. Image AFM d'un réseau aléatoire de nanotubes déposé et photographie du film flexible transparent. Extrait avec la permission de Nanoletters 5 (4) (2005) 757-760, Ref. [18a]. Copyright (2005) American Chemical Society.

Fig. 4. AFM image of a random network of nanotubes deposited on a flexible, transparent photographic film. Published with permission of Nanoletters 5 (4) (2005) 757-760, Ref. [18a]. Copyright (2005) of the American Chemical Society.

lité potentielle de tels transistors. En effet, la mobilité estimée pour les transistors publiés dans ce premier papier était déjà de l'ordre de 10 ce qui à l'époque représentait une première mondiale.

Des études plus poussées ont ensuite été publiées, notamment par les groupes de John Rogers et de George Grüner avec l'étude de la transparence, de la conductivité et du ratio on-off de ces composants en fonction de la densité du réseau en nanotube et notamment vis-à-vis du seuil de percolation. Les performances obtenues ne sont cependant pas encore d'une qualité suffisante pour permettre une application transistor de ces composants pour les écrans plats, et ce notamment au niveau de leur conductance et modulation (Fig. 4) [18].

\subsection{Méthodes de synthèse}

De nombreuses méthodes de croissance, directement sur le substrat, de tels réseaux ont été publiées et ce n'est pas l'objet de cet article d'en faire une liste exhaustive. Parmi tous ces travaux on peut cependant citer l'utilisation comme catalyseur de croissance par le groupe de Hong Jie Dai à l'Université de Stanford d'une protéine à cluster de fer, la ferritine [19], ou encore l'utilisation de polyoxometallates [20]. Ce dernier article rapporte probablement la méthode de croissance 

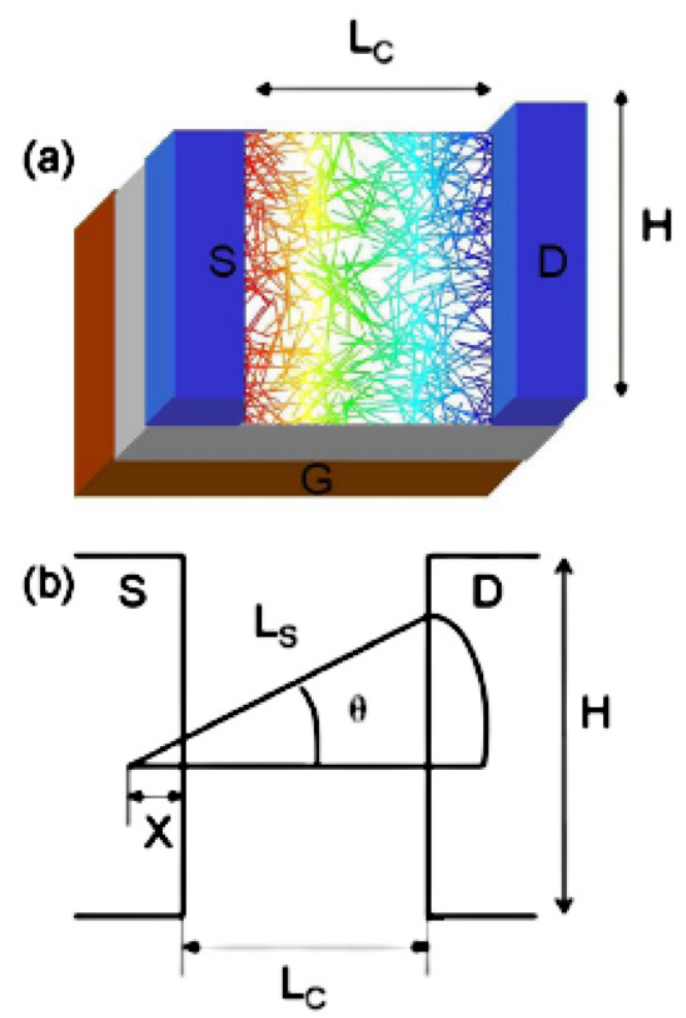

Fig. 5. a) Un transistor à réseau aléatoire de nanotubes ( $L_{C}:$ longueur du canal; $H$ : largeur du canal; $\mathrm{S}:$ source; $\mathrm{D}:$ drain); le code de couleur du réseau reflète de la distribution typique du potentiel électrique du réseau pour un canal $L_{C}=3 \mu \mathrm{m}, H=4 \mu \mathrm{m}, L_{S}=2 \mu \mathrm{m}, c_{i j}=5: 0$, and $r=3,5 \mu \mathrm{m}^{2}$. b) Nomenclature utilisée. Extrait avec la permission de Physics Review Letters 95 (2005) 066802, Ref. [26]. Copyright (2005) de l'American Physical Society.

Fig. 5. a) A thin-film network transistor with channel length $L_{C}$, channel width $H$, and individual tube length $L_{S}$. Source (S), drain (D), and gate (G) are also indicated; the color code of the network reflects the typical potential distribution in a nanotube network for a channel with $L_{C}=3 \mu \mathrm{m}, H=4 \mu \mathrm{m}$, $L_{S}=2 \mu \mathrm{m}, c_{i j}=5: 0$, and $r=3.5 \mu \mathrm{m}^{2}$. b) Nomenclature for bridging tube calculation. Published with permission from Physics Review Letters 95 (2005) 066802, Ref. [26]. Copyright (2005) of the American Physical Society.

permettant la croissance d'un réseau ayant une dispersion de diamètres des CNTs monoparois la plus monodisperse et ce à partir d'un polyoxometallate relativement facile à synthétiser.

D'autres groupes se sont concentrés sur des méthodes de transfert ou de dépôt par voies liquides. On peut notamment citer les travaux du groupe de Robert Haddon qui utilise des nanotubes purifiés et/ou fonctionnalisés (commercialisés par Carbon Solutions, Inc.) et qui les dépose par spray [21]. Le groupe de V. Tsukruk dépose les nanotubes par une méthode dérivée du dip-coating à partir de solution mésogènes à base de nanotubes qui permet d'obtenir des réseaux à des degrés d'orientation modulable [22]. Quant au groupe de J.A. Rogers, il dépose les nanotubes par un procédé de transfert à partir d'un tampon [23]. Si toutes ces méthodes permettent effectivement d'obtenir des transistors, leurs propriétés électroniques sont encore très médiocres, notamment d'un point de vue ratio on/off, conductance et hystérésis. A cet effet, l'utilisation de nanotubes triés devrait permettre d'améliorer certaines de ces propriétés [24]. Une société, NanoIntegris, Inc., spin off de l'Université de Northwestern, a notamment été créée récemment pour la commercialisation de nanotubes triés. Cette société devrait mettre ses produits sur le marché vers la fin 2008, d'après son fondateur, Mark C. Hersam de l'Université de Northwestern [25].

\section{Modélisation}

Il a fallu attendre 2005 pour voir apparaitre les premiers travaux de modélisation de ces réseaux aléatoire qui se sont révélés être d'excellents modèles d'études théoriques. Le groupe leader dans ce domaine est celui de M.A. Alam de l'Université de Purdue. Dans un premier article, un modèle a été développé permettant de simuler le comportement de ces réseaux en fonction de la densité des tubes et de leur longueur (Fig. 5) [26].

Le modèle a par la suite été plus amplement développé pour prendre en compte divers effets comme celui du substrat et des charges des surface trappées [27], ou encore de l'influence d'une orientation partielle des nanotubes au sein du réseau [28]. Une dernière étude à porté sur la simulation de leurs propriétés de conduction thermique [29]. Notons que ce modèle leur a permis de démontrer théoriquement puis expérimentalement la faisabilité d'augmenter de cinq ordres de grandeurs 


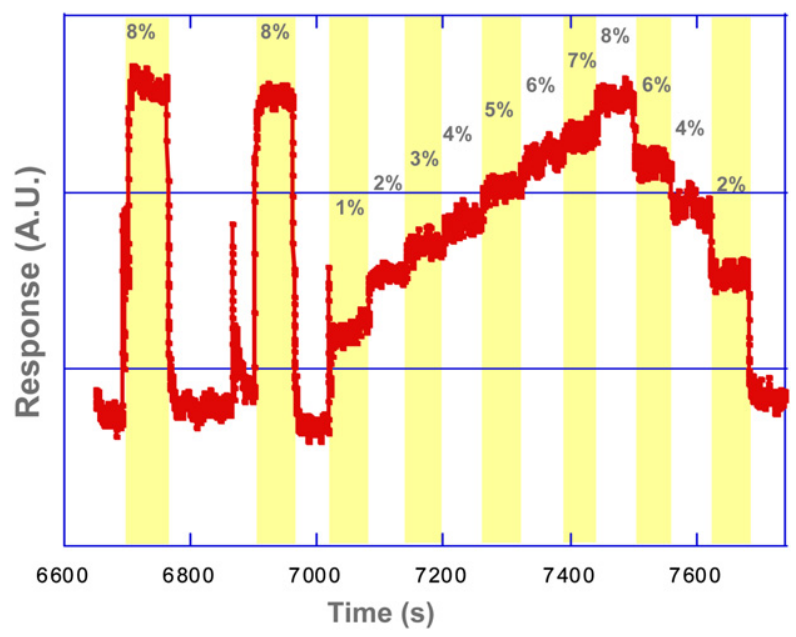

Fig. 6. Réponse en mode capacitif d'un senseur à réseau aléatoire de nanotube au gas d'anesthésie Sevoflurane dans le domaine typique d'utilisation de ce composant lors d'une procédure d'anesthésie (0 à $8 \%$ ). D'après la Réf. [37].

Fig. 6. Response in capacitance mode of a random network nanotube sensor to the anaesthetic gas Sevoflurane in a typical use of this during anaesthesia (0 to $8 \%$ ). From Ref. [37].

la conductance par une optimisation théorique du taux d'orientation des nanotubes sans que cela se fasse au détriment de la modulation [30].

\section{Applications}

\subsection{Senseurs chimiques}

La sensibilité aux facteurs extérieurs des transistors à nanotubes a été observée très tôt [31]. En première approximation, on peut dire que lorsque des molécules donneuses ou accepteuses d'électrons viennent à proximité d'un nanotube, il s'ensuit un transfert d'électron qui peut être mesuré directement au niveau de la caractéristique électronique du transistor. Dans le détail, il semble que la sensibilité puisse provenir de différentes contributions : (i) le dopage peut s'effectuer au niveau de la barrière de Schottky qui existe à l'interface des nanotubes semiconducteurs et des métaux utilisés pour les contacts électriques (l'or est souvent utilisé) [32]. Le palladium a ainsi été introduit pour éviter cet effet [33]; (ii) la détection peut s'effectuer au niveau du corps des nanotubes eux-mêmes [34]; (iii) enfin elle peut s'effectuer au niveau des défauts des nanotubes [35].

Cette sensibilité semble avoir été conservée lors du passage de systèmes à nanotubes unique vers ceux à réseaux aléatoires de SWNTs. Un mécanisme supplémentaire de détection est alors à étudier où la détection s'effectue au niveau des jonctions nanotubes-nanotubes.

Le mode de détection électrique peut se faire directement en mode résistif, en courant continu ou alternatif ou par la mesure d'un paramètre spécifique de la caractéristique IVg (tension de seuil, impédance à une valeur de la tension de grille donnée), etc. Dans le cadre de la mesure d'impédance, une autre méthode publiée par le groupe d'Eric Snow, est d'utiliser le réseau aléatoire comme l'une des deux électrodes d'un condensateur, l'autre étant la grille en face arrière [36]. Cette méthode est complémentaire de la précédente car elle permet la détection de molécule à nuage électronique polarisable. Cette stratégie a permis à Nanomix le développement d'une stratégie de détection des molécules utilisées dans le domaine de l'anesthésie, qui sont en général riche en halogène et sont facilement polarisables (Fig. 6) [37].

Ces transistors peuvent aussi être utilisés immergés, en solution aqueuse ou non, et notamment avec l'utilisation, non plus d'une grille en face arrière, mais d'une grille immergée, ce qui permet de réduire les voltages de grille nécessaires d'un ordre de grandeur [38]. Cette configuration est très utile pour le développement de senseurs en phase aqueuse et notamment de biosenseurs (Fig. 7) [39,40].

Si de tels FETs présentent une bonne sensibilité à de nombreuses molécules, il s'agit aussi de leur talon d'Achille car de tels structures représentent de très bons transducteurs mais de fait elles ne sont donc pas spécifiques. Or, la plupart des cahiers des charges de senseurs commerciaux nécessitent l'absence de faux positifs et négatifs qui tous deux peuvent avoir de lourdes conséquences. Il s'agit donc de développer des stratégies de fonctionnalisation de ces senseurs permettant de réaliser une bonne spécificité dans l'environnement prévu par le cahier des charges [41]. En cela les senseurs à CNT ne diffèrent pas des autres types de senseurs, et le coût associé au développement de ces stratégies ne doit pas être sous estimé. Ainsi pour la réalisation du détecteur à Hydrogène commercialisé par Nanomix en mai 2005 [42] c'est un alliage à base de palladium qui a permis d'obtenir une bonne spécificité du senseur [43]. Dans le cas du développement d'un détecteur de $\mathrm{CO}_{2}$ pour les applications dans le domaine de la capnographie (mesure du $\mathrm{CO}_{2}$ dans l'haleine) c'est une 


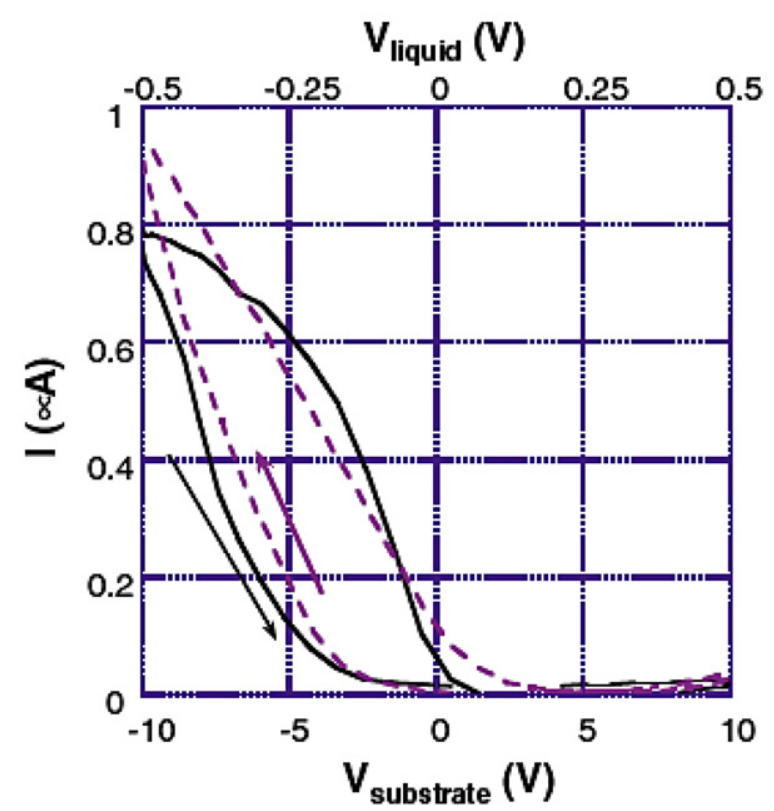

Fig. 7. Caractéristique électronique d'un NT-FET dans l'air (ligne continue) en utilisant une grille face arrière et dans l'eau (ligne discontinue) en utilisant une grille immergée. Les flèches indiquent le sens du balayage. Notez les différences d'échelles des abscisses pour ces deux configurations. Extrait (Fig. 2) avec la permission de Physical Review Letters 91 (21) (2003) 218301, Ref. [39]. Copyright (2003) by the American Physical Society.

Fig. 7. Electronic characteristics of a NT-FET in air (solid line) using a grid as the back face and in water (dashed line) using a submerged grid. The arrows indicate the scanning direction. Note the different abscissa scales for 2 configurations. Reprinted (Fig. 2) with permission from Physical Review Letters 91 (21) (2003) 218301, Ref. [39]. Copyright (2003) by the American Physical Society.

formulation complexe à base de polyéthylèneimine qui a joué ce rôle [44]. D’autres stratégies ont été développées comme la mise en place de barrières de diffusions [45].

Lorsqu'il n'est pas possible de trouver une fonctionnalisation induisant une haute spécificité, une autre approche est possible. Dans ce cas, on utilise plusieurs senseurs, chacun ayant une fonctionnalisation propre. Si les matériaux de fonctionnalisation sont bien choisis, c'est la réponse collective qui permet de différencier les molécules les unes des autres. Cette approche est celle utilisée dans les nez artificiels [46].

Au bilan, de nombreux travaux sont maintenant dans la littérature sur le sujet, et qui pour la plupart en restent à l'observation d'une variation de comportement lors de l'exposition du composant à un stimulus extérieur (lumière [47], gas [48], molécules en solution, bio-molécules [40,49] - glucose, ADN [50], protéines [51], dérivés benzéniques [52], et même mécaniques).

Par contre, l'utilisation de ce type de composants dans des senseurs commerciaux nécessite de lourds investissements (y compris dans le développement des bancs de tests) afin de les amener sur le marché sous une forme qui réponde au cahier des charges et à l'attente du client. Seuls les produits à haut volume et à très forte valeur ajoutée peuvent être étudiés en vue d'une possible production même si, en principe, cette structure peut se voir appliquée dans de nombreux domaines. Dans cette optique, Nanomix a développé avec succès jusqu'au stade du prototype produit un détecteur d'ammoniaque fonctionnant en atmosphère résiduel d'ammoniaque ce qu'aucun senseur commercial actuel ne permet de faire. Nanomix recherche un distributeur pour la fabrication et la commercialisation de ce produit (Fig. 8).

Les efforts commerciaux de Nanomix se concentrent à l'heure actuelle sur la mesure de NO dans l'haleine comme traceur d'asthme, une maladie qui touche 20 millions d'américains, une application issue d'une collaboration avec l'Université de Pittsburg [53].

\subsection{Composants électroniques}

De nombreux brevets ont été pris par la société Nantero, Inc. (Woburn, Massachusetts) qui s'est initialement lancée sur le développement d'une mémoire réinscriptible et capable de conserver des données sans alimentation électrique, ce qui en fait potentiellement une mémoire universelle, utilisable dans une multitude d'applications. Ce type de mémoire, appelée NRAM par Nantero, exploite la stabilité bimodale d'une matrice de nanotubes de carbone déposée dans un sillon préalablement gravé [54]. Ce réseau positionné généralement au dessus d'un via peut se déformer sous l'application d'un champ électrique, et ainsi venir au contact d'une électrode située en dessous du réseau. Il s'agit là d'un nanoswitch qui peut être intégré dans des composants et architectures variés tels que des mémoires, switchers, émetteurs de lumière, récepteurs, transistors électromécaniques, circuits logiques (Fig. 9). En avril 2006, Nantero, Inc. a annoncé avoir fabriqué et testé avec succès un commutateur de mémoire en technologie $22 \mathrm{~nm}$ [55]. Une sortie commerciale était alors annoncée pour 2007. 


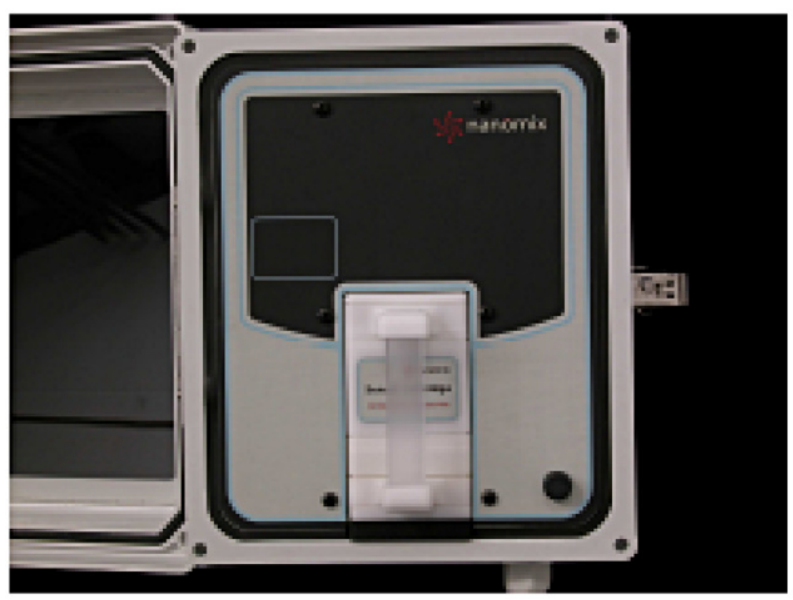

Fig. 8. Le prototype pre-production de Nanomix pour la détection de $\mathrm{NH}_{3}$ en milieu avicole (source Nanomix). Extrait avec la permission de Nanomix. Fig. 8. The Nanomix pre-production prototype for an $\mathrm{NH}_{3}$ detector to be used in bird raising (from Nanomix, with permission).

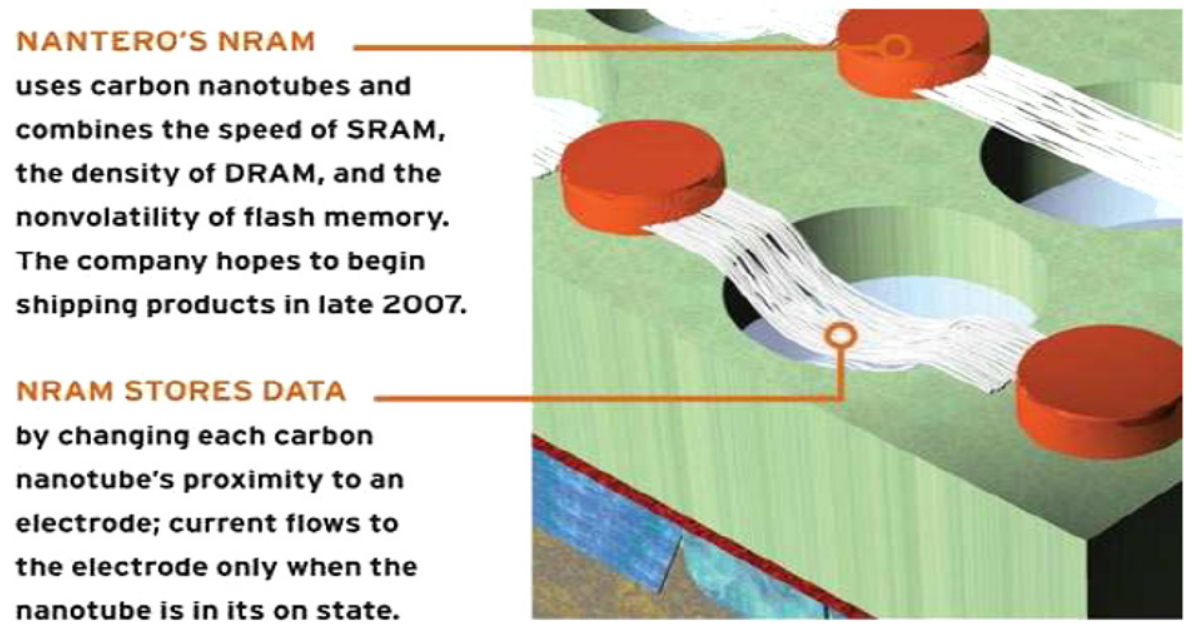

Fig. 9. Schéma décrivant l'architecture des commutateurs développés par la société Nantero. Si un potentiel adéquate est appliqué entre le faisceau de nanotubes et l'électrode situé au fond du puits, le faisceau initialement isolé (état OFF) peut être déformé et venir au contact avec l'électrode sousjacente (état ON).

Fig. 9. Drawing of the switch architecture developed by the society Nantero. If a sufficient potential is applied between the beam of nanotubes and the electrode situated at the bottom on the well, the initially isolated beam (OFF position) can de deformed and make contact with the underlying electrode (ON position).

\subsection{Film conducteur comme électrode flexible}

L'utilisation des réseaux désordonnés de nanotubes comme conducteur électronique est aussi très avancé d'un point de vue industriels.

Un premier domaine est dans le remplacement des couches de carbone amorphe utilisées dans les bandelettes de mesure du taux de glucose dans le sang. En effet, la reproductibilité de ces couches n'est pas excellente ce qui force les industriels à de coûteuses phases de calibration. Le remplacement de cette couche par un film de nanotube en réseau désordonné a été proposé et son utilisation est en cours d'étude. L'avantage principal serait une meilleure conductance ainsi qu'une meilleure reproductibilité.

Une deuxième application est étudiée par plusieurs industriels : le remplacement de l'ITO (Indium-Tin-Oxide) qui est utilisé comme coûche conductrice et transparente dans de nombreuses applications comme les écrans tactiles, les affichages cristaux liquides, les écrans souples, etc.

On peut notamment citer les travaux initiés à / breveté par Nanomix puis développés à UCLA par George Grüner qui ont étudié l'influence de la densité et épaisseur du réseau en nanotube sur la conductance et la transparence de ces films. Ces travaux ont permis la création d'une startup, Nanodym, Inc., qui a depuis étè rachetée par Arrowhead et qui est sur le 
(a)
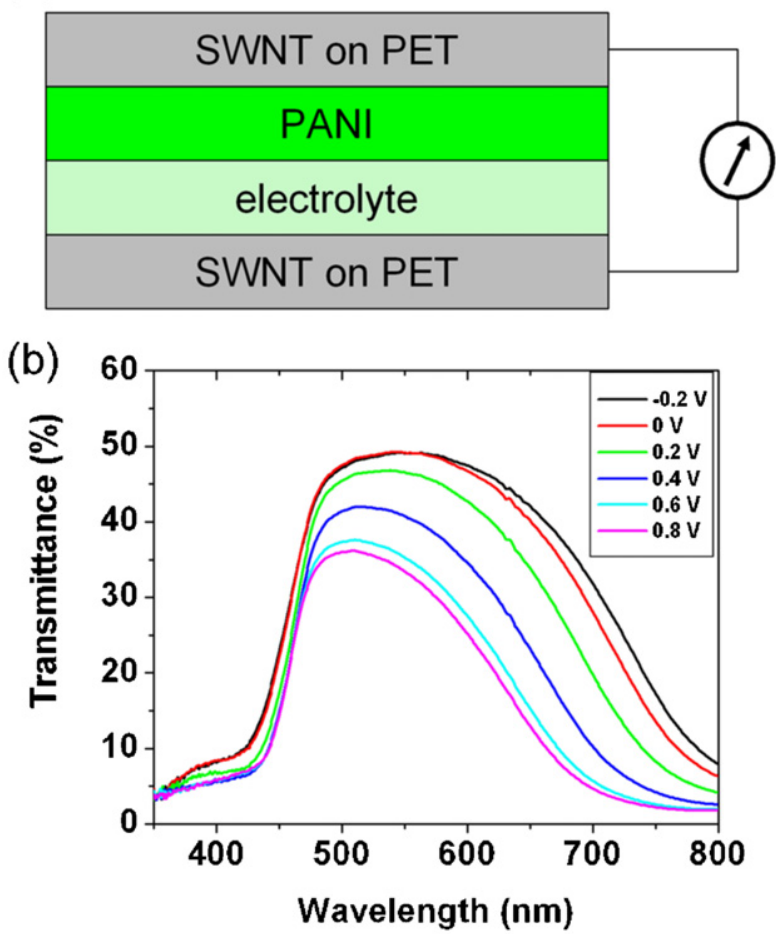

Fig. 10. a) Architecture d'une fenêtre "intelligente» dont la couleur peut être modulée; b) Transmission optique du composant pour différentes valeurs de voltage appliqué. Extrait avec la permission de Journal of Applied Physics 101 (2007) 016102. Copyright (2007) American Institute of Physics.

Fig. 10. a) Architecture of an "intelligent" window whose colour can be modulated; b) Optical transmission of this component for different values of the applied voltage. Published with permission of the Journal of Applied Physics 101 (2007) 016102. Copyright (2007) the American Institute of Physics.

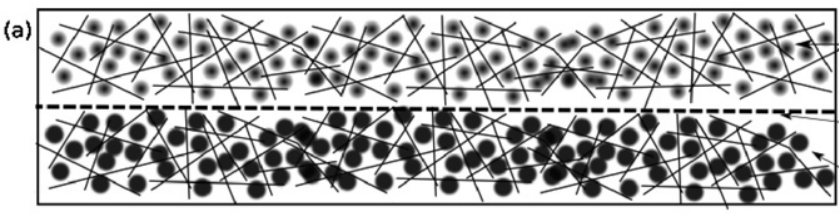

Active cathode material mixed with nanowires

Separator (+ electrolyte)

Active anode material

mixed with nanowires

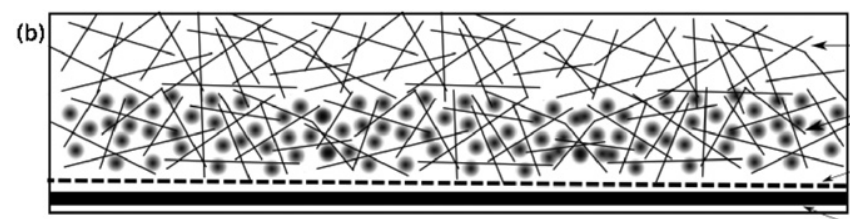

Nanowire network

(charge collector)

Active cathode material

mixed with carbon nanotubes

Separator (+ electrolyte)

Active anode material (Zinc foil),

also acts as charge collector)

Fig. 11. a) Proposition de design d'une batterie incorporant le matériau de l'électrode active et celui du collector en une seule couche; b) Design d'un démonstrateur construit à partir d'un collecteur de charge à base d'un film de nanotubes de carbones et d'un matériau actif à base d'un mélange de $\mathrm{MnO}_{2}$ et de nanofils. Extrait avec la permission du Journal Applied Physics Letters 91 (2007) 144104 (Ref. [61]); copyright (2007) de l'American Institute of Physics.

Fig. 11. a) Design proposition for a battery with an active electrode and collector formed by a single layer; b) Demonstrator design using a charge collector made from a film of carbon nanotubes and an active material based on a mixture of $\mathrm{MnO}_{2}$ and nanowires. Published with permission of the Journal Applied Physics Letters 91 (2007) 144104 (Ref. [61]); copyright (2007) of the American Institute of Physics.

point de mettre sur le marché des films PET (polyéthylèneterephthalate) à couche conductrice à base de réseaux aléatoires de nanotubes de carbone. Ces films sont destinés à être intégrés dans des écrans tactiles [56].

D'autres travaux semblent indiquer que ces films pourraient aussi être intégrés un jours dans des OLED [57], cellules photovoltaïques [58], ou encore comme électrode dans des applications électrochromiques de type écrans flexibles (Fig. 10) [59]. Pour ces applications, la combinaison de la haute transparence, la faible résistance électrique des couches, leur caractère robuste mécaniquement, leur stabilité environnementale et la facilité de leur dépôt de façon industrielle sont mis en avant comme atouts clés. 
Enfin, la société Nantero a récemment annoncé une collaboration avec HP pour la réalisation d'électronique souple combinant leur savoir faire en dispersion des nanotubes et celui d'HP en technologie Ink-Jet [60].

Une dernière application à récemment été publiée pour leur utilisation comme collecteur de charge, ainsi qu'intégré dans des composites dans l'anode et la cathode dans l'architecture de batteries souples (Fig. 11) [61].

\subsection{Les acteurs industriels}

La demande mondiale en nanotubes de carbone augmente rapidement du fait de la maturation de ses applications. En effet, le marché global des nanotubes de carbone devrait dépasser les \$1.9 milliards en 2010 d'après un récent rapport de Global Industry Analysts, Inc. [62]. On commence d'ailleurs à voir les premières consolidations s'effectuer avec, par exemple, l'acquisition par Arrowhead de Unidym Inc et plus récemment de Carbon Nanotechnologies, Inc., la société initialement créée par le lauréat du prix Nobel, Prof. Richard Smalley de l'Université de Rice, USA.

Une liste des acteurs commerciaux principaux dans le domaine des nanotubes est donnée dans le Tableau 1.

\section{Tableau 1}

Les acteurs commerciaux principaux dans le domaine des nanotubes.

Table 1

The principal commercial actors in nanotubes.

\begin{tabular}{|c|c|c|}
\hline Organisation & Méthode de production / Produit & Status \\
\hline \multicolumn{3}{|l|}{ Matériaux } \\
\hline $\begin{array}{l}\text { SouthWest NanoTechnologies, Norman, Okla. } \\
\text { (www.swnano.com) }\end{array}$ & Production par la technique de CVD & Production \\
\hline Arkema & Ouverture d'un plan de 400 tonnes/an en 2010 & Production \\
\hline Ahwahnee Technology (USA) & Pas d'information sur la méthode de production & Production \\
\hline Apex Nanomaterials (USA) & Production par la technique de l'arc électrique & Production \\
\hline Arry International Group, Ltd. (Germany) & $\begin{array}{l}\text { Production par la technique de CVD; purification; } \\
\text { fonctionalisation }\end{array}$ & Production \\
\hline Bayer MaterialScience AG (Germany) & Pas d'information sur la méthode de production & Production \\
\hline BuckyUSA (USA) & Production par la technique de l'arc électrique & Production \\
\hline CarboLex, Inc. (USA) & $\begin{array}{l}\text { Productions par les techniques de l'arc électrique et de } \\
\text { dépôt en phase vapeur (CVD) }\end{array}$ & Production \\
\hline Carbon Nanotechnologies, Inc. (USA) & $\begin{array}{l}\text { Productions par les techniques de CVD à partir de } \\
\text { ferrocène et monoxide de carbone et ablation laser }\end{array}$ & $\begin{array}{l}\text { Production (racheté par } \\
\text { Arrowhead, avril 2007) }\end{array}$ \\
\hline Carbon NT\&F 21 (Austria) & Pas d'information sur la méthode de production & Production \\
\hline Carbon Solutions, Inc. (USA) & $\begin{array}{l}\text { Production par la technique de l'arc électrique, } \\
\text { purification et modification chimiques }\end{array}$ & Production \\
\hline Catalytic Materials LLC (USA) & Production par la technique de CVD & Production \\
\hline Cheap Tubes, Inc. (USA) & Purification; fonctionnalisation & Distributeur \\
\hline Chengdu Organic Chemicals Co., Ltd. (China) & Production par la technique de CVD & Production \\
\hline Heji, Inc. (Hong Kong) & $\begin{array}{l}\text { Pas d'information sur la méthode de production, } \\
\text { probablement CVD; purification; fonctionnalisation }\end{array}$ & Production \\
\hline Helix Material Solutions (USA) & Production par la technique de CVD & Production \\
\hline Idaho Space Materials, Inc. (USA) & $\begin{array}{l}\text { Production par la technique de l'arc électrique, sans } \\
\text { catalyseur métallique }\end{array}$ & Production \\
\hline Iljin Nanotech Co., Ltd. (Korea) & $\begin{array}{l}\text { Productions par les techniques de l'arc électrique et de } \\
\text { dépôt en phase vapeur (CVD) }\end{array}$ & Production \\
\hline M.E.R. Corp. (USA) & $\begin{array}{l}\text { Productions par les techniques de l'arc électrique et de } \\
\text { dépôt en phase vapeur (CVD) }\end{array}$ & Production \\
\hline MicrotechNano (USA) & $\begin{array}{l}\text { Pas d'information sur la méthode de production, } \\
\text { probablement CVD }\end{array}$ & Production \\
\hline Nano-C (USA) & Production par la technique de CVD & Production \\
\hline NanoCarbLab (Russia) & $\begin{array}{l}\text { Production par la technique de l'arc électrique, } \\
\text { purification et modification chimiques }\end{array}$ & Production \\
\hline Nanocomp Technologies, Inc. (USA) & Longs nanotubes par CVD & Production \\
\hline Nanocs (USA) & $\begin{array}{l}\text { Productions par les techniques de l'arc électrique et de } \\
\text { dépôt en phase vapeur (CVD); purification et } \\
\text { modification chimiques }\end{array}$ & Production \\
\hline Nanocyl S.A. (Belgium) & Production par la technique de CVD & Production \\
\hline Nanolntegris (www.nanointegris.com) & $\begin{array}{l}\text { Nanotubes triés par fonctionalisation suivit de } \\
\text { centrifugation successives (spinoff de l'Université de } \\
\text { Northwestern) }\end{array}$ & $\begin{array}{l}\text { Production prévue pour } \\
\text { fin } 2008\end{array}$ \\
\hline NanoLab (USA) & $\begin{array}{l}\text { Production par la technique de CVD et Plasma enhanced } \\
\text { CVD (PE-CVD pour les films de CNTs alignés } \\
\text { verticalement) }\end{array}$ & Production \\
\hline Nanoledge (France) & $\begin{array}{l}\text { A arrêté la production de CNT par la méthode de l'arc } \\
\text { électrique, se focalise sur la formulation de } \\
\text { composites pour applications à haute valeur ajoutée }\end{array}$ & $\begin{array}{l}\text { Production et R\&D (en } \\
\text { cours de délocalisation au } \\
\text { Canada) } \\
\quad \text { (suite à la page suivante) }\end{array}$ \\
\hline
\end{tabular}


Tableau 1 (suite)

\begin{tabular}{|c|c|c|}
\hline Organisation & Méthode de production / Produit & Status \\
\hline Nanostructured \& Amorphous Materials, Inc. (USA) & Production par la technique de CVD & Production \\
\hline NanoTechLabs, Inc. (USA) & Production par la technique de CVD & Production \\
\hline Nanothinx S.A. (Greece) & Production par la technique de CVD & Production \\
\hline n-Tec (Norway) & Production par la technique de l'arc électrique & Production \\
\hline Raymor Industries, Inc. (Canada) & Production par la technique de la torche à plasma & Production \\
\hline Rosseter Holdings, Ltd. (South Cyprus) & $\begin{array}{l}\text { Production par la technique de l'arc électrique à bas } \\
\text { voltage en phase liquide }\end{array}$ & Production \\
\hline SES Research (USA) & Production par la technique de CVD & Production \\
\hline Shenzhen Nanotechnologies Co., Ltd. (China) & $\begin{array}{l}\text { Pas d'information sur la méthode de production, } \\
\text { probablement CVD }\end{array}$ & Production \\
\hline Sun Nanotech Co., Ltd. (China) & Production par la technique de CVD & Production \\
\hline Thomas Swan \& Co., Ltd. (England) & Production par la technique de CVD, hauts volumes & Production \\
\hline Xintek, Inc. (USA) & Production par la technique de CVD & Production \\
\hline 21st Century NanoTechnologies, Inc. (China) & Production par la technique de CVD & Production \\
\hline Ironbark Composites (Australia) & $\begin{array}{l}\text { Pas d'information sur la méthode de production, } \\
\text { probablement CVD }\end{array}$ & $\begin{array}{l}\text { MWNTs, production } \\
(\$ 500 / \mathrm{kg} \text { au } 22 / 01 / 2008)\end{array}$ \\
\hline Films tranparents & & Production \\
\hline $\begin{array}{l}\text { Battelle Memorial Institute, Columbus, Ohio } \\
\text { (www.battelle.org) }\end{array}$ & Dépôt de couche mince transparente & $\mathrm{R} \& \mathrm{D}$ \\
\hline Eikos, Franklin, Mass. (www.eikos.com) & Encres pour dépôts de films conducteurs; formulation & $\begin{array}{l}\text { Production, } \\
\text { développement de } \\
\text { produits et R\&D }\end{array}$ \\
\hline Eastman Kodak, Rochester, N.Y. (www.kodak.com) & Dépôt de couche mince transparente & R\&D, prototype \\
\hline Unidym, Menlo Park, Calif. (www.unidym.com) & $\begin{array}{l}\text { Films transparents conducteurs pour écrans tactiles, } \\
\text { cellules photovoltaïques et diodes } \\
\text { électroluminescentes }\end{array}$ & $\begin{array}{l}\text { Produits en cours de } \\
\text { développement, premier } \\
\text { produit attendu courant } \\
2008 \text { (racheté par } \\
\text { Arrowhead en 2006) }\end{array}$ \\
\hline \multicolumn{3}{|l|}{ Composants } \\
\hline DuPont, Wilmington, Del. (www.dupont.com) & Electronique transparente & R\&D \\
\hline IBM, Armonk, N.Y. (www.ibm.com) & Transistors et interconnect & R\&D \\
\hline Intel, Santa Clara, Calif. (www.intel.com) & Transistors et interconnect & R\&D \\
\hline Motorola, Schaumburg, Ill. (www.motorola.com) & Senseurs chimiques et biochimiques & Prototype \\
\hline Nanomix, Emeryville, Calif. (www.nano.com) & Senseurs chimiques et biochimiques & $\begin{array}{l}\text { Production, prototypes, } \\
\text { R\&D }\end{array}$ \\
\hline Nantero, Woburn, Mass. (www.nantero.com) & NRAM ; encre et formulation ; électronique flexible & Prototype \\
\hline Samsung, Seoul, South Korea (www.samsung.com) & Ecrans, interconnect & $\mathrm{R} \& \mathrm{D}$ \\
\hline Unidym (voir au-dessus) & $\begin{array}{l}\text { Electronique imprimée pour écrans, écrans tactiles, } \\
\text { OLED, baterrie et électronique souple }\end{array}$ & $\begin{array}{l}\text { Prototypes } \\
\text { pre-production; proof of } \\
\text { concept }\end{array}$ \\
\hline Xintek, Inc. (USA) & Cathode, tube à rayons $\mathrm{X}$, pointes $\mathrm{AFM}$ & Production \\
\hline
\end{tabular}

\section{Conclusions}

Les réseaux aléatoires à nanotubes de carbone représentent la première réponse des industriels au problème de la polydispersité en propriété physiques des transistors à nanotubes. Ils vont permettre l'intégration des nanotubes dans des produits complexes à haute valeur ajoutée comme l'illustre la mise sur le marché des premiers produits électroniques à base de nanotubes et le grand nombre d'applications en cours de développement. Le remplacement de l'ITO par les nanotubes représenterait notamment une percée majeure, à forts volumes, pour l'obtentions de films plastiques conducteurs à intégrer dans les écrans tactiles qui ne devraient pas présenter de problème de fatigue mécanique. Une amélioration importante des propriétés électroniques des films déposés par voie humide est cependant nécessaire pour que ces réseaux percent dans le milieu des composants électroniques flexibles de type transistor à effet de champ.

\section{Remerciements}

J.C.G. tient à remercier ses anciens collègues de Nanomix et l'Observatoire des micro- et nanotechnologies pour son autorisation de publication de cette monographie.

\section{Références}

[1] M. Monthioux, V.L. Kuznetsov, Who should be given the credit for the discovery of carbon nanotubes? Carbon 44 (2006) 1621-1623 (Editorial).

[2] S. Iijima, Nature 354 (1991) 56-58.

[3] D.S. Bethune, et al., Cobalt-catalysed growth of carbon nanotubes with single-atomic-layer walls, Nature 363 (1993) 605-607, doi:10.1038/363605a0.

[4] a) S.J. Tans, A.R.M. Verschueren, C. Dekker, Nature 393 (1998) 49;

b) R. Martel, T. Schmidt, H.R. Shea, T. Hertel, P. Avouris, Appl. Phys. Lett. 73 (1998) 2447.

[5] a) J. Kong, H.T. Soh, A.M. Cassell, C.F. Quate, H. Dai, Nature 395 (1998) 878;

b) A.M. Cassell, N.R. Franklin, T.W. Tombler, E.M. Chan, J. Han, H. Dai, J. Am. Chem. Soc. 121 (1999) 7975-7976. 
[6] G. Tennent, et al., US Patent No. 5,578,543.

[7] P.G. Collins, M.S. Arnold, P. Avouris, Engineering carbon nanotubes and nanotube circuits using electrical breakdown, Science 292 (2001) $706-709$.

[8] J.-C. Gabriel, K. Bradley, P. Collins, Dispersed growth of nanotubes on a substrate, WO 2004040671A2.

[9] J.-C.P. Gabriel, Large scale production of carbon nanotube transistors: A generic platforms for chemical sensors, in: Mat. Res. Soc. Symp. Proc. vol. 762, 2003, Q.12.7.1

[10] E.S. Snow, J.P. Novak, P.M. Campbell, D. Park, Random networks of single-wall carbon nanotubes as an electronic material, Appl. Phys. Lett. 82 (2003) 2145-2147.

[11] J.P. Novak, E.S. Snow, E.J. Houser, D. Park, J.L. Stepnowoski, R.A. McGill, Nerve agent detection using networks of single-wall carbon nanotubes, Appl. Phys. Lett. 83 (2003) 4026-4028.

[12] M.S. Fuhrer, J. Nygård, L. Shih, M. Forero, Young-Gui Yoon, M.S.C. Mazzoni, Hyoung Joon Choi, Jisoon Ihm, Steven G. Louie, A. Zettl, Paul L. McEuen, Science 288 (5465) (2000) 494-497.

[13] a) Y. Fan, B.R. Goldsmith, P.G. Collins, Identifying and counting point defects in carbon nanotubes, Nature Mater. 4 (2005) 906; b) D. Kingrey, O. Khatib, P.G. Collins, Electronic fluctuations in nanotube circuits and their sensitivity to gases and liquids, Nano Lett. 6 (2006) 1564.

[14] M. Ohishi, M. Shiraishi, K. Ochi, Y. Kubozono, H. Kataura, Appl. Phys. Lett. 89 (2006) 203505.

[15] K. Bradley, J. Cumings, A. Star, J.-C.P. Gabriel, G. Grüner, Influence of mobile ions on nanotube based FET devices, Nano Lett. 3 (5) (2003) 639-641.

[16] W.-K. Hong, C. Lee, D. Nepal, K.E. Geckeler, K. Shin, T. Lee, Radiation hardness of the electrical properties of carbon nanotube network field effect transistors under high-energy proton irradiation, Nanotechnology 17 (2006) 5675-5680.

[17] a) K. Bradley, J.-C.P. Gabriel, G. Grüner, Flexible nanotube transistors, Nano Lett. 3 (10) (2003) 1353;

b) P.N. Armitage, K. Bradley, J.-C.P. Gabriel, G. Gruner, Flexible nanostructure electronic devices, United States Patent 20050184641 A1.

[18] a) E. Artukovic, M. Kaempgen, D.S. Hecht, O. Roth, G. Grüner, Transparent and flexible carbon nanotube transistors, Nano Lett. 5 (4) (2005) 757-760; b) L. Hu, D.S. Hecht, G. Grüner, Percolation in transparent and conducting carbon nanotube networks, Nano Lett. 4 (12) (2004) 2513-2517; c) Q. Cao, S.-H. Hur, Z.-T. Zhu, Y. Sun, C. Wang, M.A. Meitl, M. Shim, J.A. Rogers, Adv. Mater. 18 (2006) 304-309.

[19] Y. Li, W. Kim, Y. Zhang, M. Rolandi, D. Wang, H. Dai, J. Phys. Chem. B 105 (2001) 11424-11431.

[20] J. Am. Chem. Soc. 124 (46) (2002) 13688-13689, doi:10.1021/ja0274958.

[21] E. Bekyarova, M.E. Itkis, N. Cabrera, B. Zhao, A. Yu, J. Gao, R.C. Haddon, Electronic properties of single-walled carbon nanotube networks, J. Am. Chem. Soc. 127 (2005) 5990-5995.

[22] H. Ko, V.V. Tsukruk, Liquid-crystalline processing of highly oriented carbon nanotube arrays for thin-film transistors, Nano Lett. 6 (2006) 1443-1448.

[23] M.A. Meitl, Y. Zhou, A. Gaur, S. Jeon, M.L. Usrey, M.S. Strano, J.A. Rogers, Solution casting and transfer printing single-walled carbon nanotube films, Nano Lett. 4 (9) (2004) 1643-1647.

[24] a) M.S. Arnold, A.A. Green, J.F. Hulvat, et al., Sorting carbon nanotubes by electronic structure using density differentiation, Appl. Phys. Lett. 90 (2007) 233108;

b) M.S. Arnold, A.A. Green, J.F. Hulvat, et al., Nature Nanotechnology 1 (1) (2006) 60-65.

[25] M.C. Hersam, communication personnelle, février 2008.

[26] S. Kumar, J.Y. Murthy, M.A. Alam, Phys. Rev. Lett. 95 (2005) 066802.

[27] S. Kumar, N. Pimparkar, J.Y. Murthy, M.A. Alam, Appl. Phys. Lett. 88 (2006) 123505.

[28] C. Kocabas, N. Pimparkar, O. Yesilyurt, S.J. Kang, M.A. Alam, J.A. Rogers, Nano Lett. 7 (5) (2007) 1195-1202.

[29] a) S. Kumar, M.A. Alam, J.Y. Murthy, Appl. Phys. Lett. 90 (2007) 104105; b) S. Kumar, M.A. Alam, J.Y. Murthy, J. Heat Transfer 129 (2007) 500-508.

[30] N. Pimparkar, C. Kocabas, S.J. Kang, J. Rogers, M.A. Alam, IEEE Electron Dev. Lett. 28 (7) (2007) 593.

[31] P.G. Collins, K. Bradley, Zettl A. Ishigami, Science 287 (2000) 1801.

[32] S. Heinze, J. Tersoff, R. Martel, V. Derycke, J. Appenzeller, Ph. Avouris, Phys. Rev. Lett. 89 (2002) 106801.

[33] A. Javey, J. Guo, Q. Wang, M. Lundstrom, H. Dai, Nature 424 (2003) 654-657.

[34] K. Bradley, et al., Appl. Phys. Lett. 83 (18) (2003) 3821.

[35] J.A. Robinson, E.S. Snow, S.C. Badescu, et al., Role of defects in single-walled carbon nanotube chemical sensors, Nano Lett. 6 (8) (2006) 1747-1751.

[36] E. Snow, et al., Science 307 (2005) 1942.

[37] J.-C.P. Gabriel, V. Joshi, J.L. Passmore, S. Skarupo, A. Star, C. Valcke, United States Patent Application US 20080021339.

[38] a) M. Krüger, M.R. Buitelaar, T. Nussbaumer, C. Schönenberger, L. Forró, Appl. Phys. Lett. 78 (2001) 1291-1293; b) S. Rosenblatt, Y. Yaish, J. Park, J. Gore, V. Sazonova, P.L. McEuen, Nano Lett. 2 (2002) 869-872.

[39] K. Bradley, J.C.P. Gabriel, M. Briman, A. Star, G. Grüner, Phys. Rev. Lett. 91 (21) (2003) 218301.

[40] B.L. Allen, P.D. Kichambare, A. Star, Carbon nanotube field-effect-transistor-based biosensors, Adv. Mater. 19 (11) (2007) $1439-1451$.

[41] a) A. Star, J.C. Gabriel, G. Grüner, Des exemples de stratégies de fonctionalisations peuvent être trouvées dans les brevets suivants, Nanostructure Sensor Device with Polymer Recognition Layer, S20050279987 A1;

b) J.-C.P. Gabriel, P.G. Collins, K. Bradley, G. Grüner, Modification of selectivity for sensing for nanostructure device arrays arrays, US6905655 B2; c) J.-C.P. Gabriel, S. Gandhi, A. Star, C. Valcke, Nanoelectronic glucose sensors, US20070208243 A1;

d) C.S. Joiner, J.-C.P. Gabriel, G. Grüner, A. Star, Nanotube sensor devices for DNA detection, US20070178477 A1;

e) D.M. Chang, Y.L. Chang, J.-C.P. Gabriel, V. Joshi, W. Mickelson, J. Niemann, J.L. Passmore, A. Star, C. Valcke, Carbon dioxide nanosensor, and respiratory CO2 monitors, US20070048181 A1;

f) K. Bradley, J.C. Gabriel, G. Grüner, A. Star, E. Tu, C. Valcke, System and method for electronic sensing of biomolecules, US20060228723 A1; g) J.-C.P. Gabriel, P.G. Collins, K. Bradley, G. Grüner, Modification of selectivity for sensing for nanostructure sensing device arrays, US7312095 B1.

[42] Notons que ce détecteur d'hydrogène représente le premier produit mis sur le marché intégrant des nanotubes de carbones dans un composant électronique silicium, cf. http://www.nano.com/news/archives/press_releases_and_articles/000082.html.

[43] T. Ru Han, A. Star, J.C. Gabriel, S. Skarupo, J. Passmore, P. Collins, K. Bradley, D. Olson, Nanoelectronic sensor system and hydrogen-sensitive functionalization, US20060263255 A1.

[44] United States Patent Application \#20080021339.

[45] Modification of selectivity for sensing for nanostructure sensing device arrays, United States Patent Application \#20080021339.

[46] A. Star, V. Joshi, S. Skarupo, D. Thomas, J.C.P. Gabriel, J. Phys. Chem. B 110 (2006) 21014-21020.

[47] A. Star, Y. Lu, K. Bradley, et al., Nanotube optoelectronic memory devices, Nano Lett. 4 (9) (2004) 1587-1591.

[48] E.S. Snow, F.K. Perkins, J.A. Robinson, Chemical vapor detection using single-walled carbon nanotubes, Chem. Soc. Rev. 35 (9) (2006) $790-798$.

[49] S.N. Kim, J.F. Rusling, F. Papadimitrakopoulos, Carbon nanotubes for electronic and electrochemical detection of biomolecules, Adv. Mater. 19 (2007) 3214-3228.

[50] A. Star, E. Tu, J. Niemann, J.C.P. Gabriel, C.S. Joiner, C. Valcke, Proc. Nat. Acad. Sciences 103 (4) (2006) 921-926.

[51] K. Bradley, M. Briman, A. Star, et al., Charge transfer from adsorbed proteins, Nano Lett. 4 (2) (2004) 253-256.

[52] S. Joseph, R.J. Mashl, E. Jakobsson, N.R. Aluru, Nanolett. 3 (10) (2003) 1421-1423.

[53] O. Kuzmych, B.L. Allen, A. Star, Nanotechnology 18 (2007) 375502 (7 pp.). 
[54] Voire par exemple : Nanotube-based switching elements and logic circuits, United States Patent 7138832 et US patent 6,706,402, plus de détails peuvent être trouvé sur : http://en.wikipedia.org/wiki/Nano-RAM.

[55] http://nantero.com/pdf/Release_0406.pdf.

[56] G. Grüner, communication personelle, sept. 2007.

[57] J. Li, L. Hu, L. Wang, Y. Zhou, G. Grüner, T.J. Marks, Organic light-emitting diodes having carbon nanotube anodes, Nano Lett. 6 (11) (2006) $2472-2477$.

[58] Ross A. Hatton, Anthony J. Miller, S.R.P. Silva, J. Mater. Chem. 18 (2008) 1183-1192.

[59] L. Hu, G. Grüner, D. Li, R.B. Kaner, J. Cech, J. Appl. Phys. 101 (2007) 016102.

[60] http://nantero.com/pdf/Press_ReleaseC7F35.pdf.

[61] A. Kiebele, G. Grüner, Appl. Phys. Lett. 91 (2007) 144104.

[62] Carbon nanotubes - a global strategic business report, Global Industry Analysts, Inc., USA, http://www.StrategyR.com. 